\title{
In situ Dynamic Rheological Study of Polyacrylamide during Gelation Coupled with Mathematical Models of Viscosity Advancement
}

\author{
Thibaut Savart, Caroline Dove, Brian J. Love*
}

Acrylamide dynamic viscosity has been measured in aqueous solutions. Separate rheological measurements were performed on neat resins devoid of the curing agent over a range of shear rates to yield the initial resin viscosity. The gels were also characterized by sub-ambient DSC to determine the phase structure as a function of formulation. The dynamic viscosity shows a marked sigmoidal behavior with a plateau viscosity. Mathematical interpretations of the gel time both by sigmoidal and power law models were comparable. The power law model allowed a direct determination of the gel time while the sigmoidal model yielded parameters associated with the initial viscosity, one associated with the plateau viscosity of the gel, and two time constants controlling the sharpness of the transition.

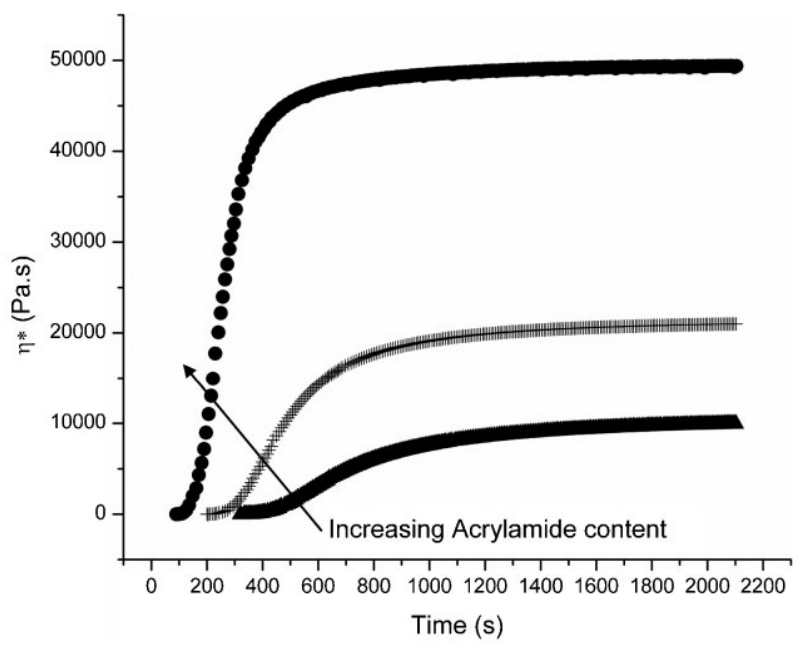

\section{Introduction}

For over 30 years, the polymerization of crosslinking acrylamide/bis acrylamide gels has generated both widespread scientific and industrial interest. Acrylamides have found use as absorbents, ${ }^{[1,2]}$ as protein separation media

T. Savart, C. Dove, B. J. Love

Department of Materials Science and Engineering, University of Michigan, Ann Arbor, MI 48109, USA

Fax: +1 734763 2013; E-mail: bjlove@umich.edu

B. J. Love

Department of Biomedical Engineering, University of Michigan,

Ann Arbor, MI 48109, USA

B. J. Love

Department of Biologic and Materials Sciences (Dentistry),

University of Michigan, Ann Arbor, MI 48109, USA using electrophoresis, ${ }^{[1,3-5]}$ as relatively non-toxic substrates to gauge cell motility, and invasively, these materials have been proposed as drug delivery platforms where a drug is formulated with the resin at the fluid stage. ${ }^{[6-8]}$ Separately, these resins have been formulated as ceramic-filled dispersions using the gel as a binder in molded preforms that are ultimately heated to remove the acrylamide yielding the sintered ceramic finished component. ${ }^{[9-13]}$

Manipulating the resin content in the gel offers significant potential to regulate protein and drug flux, as well as the mechanical stiffness of the resulting gel. Several studies have investigated the rate of acrylamide polymerization through rheological assessment; ${ }^{[1,3,6,14]}$ other research has compared tracked compositional changes and conversion using UV-Vis spectroscopy. ${ }^{[15-18]}$

Several efforts have linked the rate of acrylamide polymerization to functional properties and structure of 
the formed hydrogel. ${ }^{[16,19,20]}$ Related to this is that the polymerization is exothermic and higher polymerization temperatures are autocatalytic in advancing the rate of polymerization. The rate of polymerization affects the structures that form and this is likely to control gel performance using electrophoresis.

Separately, we have developed other nonlinear mathematical models as predictors of structure and controlled flow in reactive resins including epoxies ${ }^{[21-25]}$ and acrylates. ${ }^{[26,27]}$ Fundamentally, acrylamide polymerization and its use as a regulating structure in protein separation and electrophoresis requires a sensitivity analysis to determine what temperature and compositional parameters have the most effect on controlling the rate of polymerization and viscosity advancement. Better rheological models and interpretations might yield both more control on the structure and the performance as a separation medium. Many published studies have determined the gel time and the corresponding gel stiffness following gelation, key features tied to more fundamental studies of the structure of the formed gel. ${ }^{[28,29]}$

Resin polymerization is related to simple phenomenological models based on $n$th order kinetics where the gel time separates the flow of small molecules early on which is governed by one exponent and larger structures by a different, larger exponent. ${ }^{[25,26,30-34]}$ The gel time has been interpreted several different ways including the crossover point between the storage modulus, $G^{\prime}$, and the loss modulus, $G^{\prime \prime},{ }^{[35,36]}$ the inflection point of $G^{\prime \prime}{ }^{[37]}$ or the point where the loss tangent is frequency invariant ${ }^{[38-41]}$ using dynamic mechanical spectroscopy or rheology. Other non-linear rheological models are based on resin reactivity, ${ }^{[42,43]}$ thermodynamics, ${ }^{[44]}$ and other semi-empirical models to describe time dependent viscosity including our work on the Boltzmann sigmoidal model, ${ }^{[21,23,26,27,44]}$ according to

$$
\log \eta(t)=\log \eta_{\infty}+\frac{\log \left(\eta_{0}\right)-\log \left(\eta_{\infty}\right)}{\left(1+\mathrm{e}^{\frac{t-t_{0}}{\Delta t}}\right)}
$$

where $\eta_{0}$ corresponds to the initial viscosity of the formulated resin, $\eta_{\infty}$ correlates with a plateau viscosity either associated with maximum network density of the gel or that approaching the torque limit in the rheometer with network evolution for stiffer networks, $t_{0}$ is an induction time, corresponding to the time necessary to trigger a 50\% change in viscosity between $\log \eta_{0}$ and $\log \eta_{\infty}$ and most closely associates with the gel time, and $\Delta t$ corresponds with the period associated with the slope of $\log \eta_{0}(t)$ in the semi-log linear regime. The two time constants are shape factors and adjust the induction period before gelation and how fast one toggles between the initial and terminal viscosities.
We evaluated how the structure and dynamic viscosity are affected by curing a mixture of formulated aqueous acrylamides with concentrations varying between 9 and $18 \%$ keeping the ratio of monomer to bis crosslinker constant. The polyacrylamides were of interest due to the likelihood that the terminal viscosity of the formed gels would be a function of formulation due to their inherent increased compliance compared to other conventional resins undergoing cure. The resins were cured in the rheometer following mixing and installing a smaller mixed volume in the rheometer. Separately, the cured resins were subsequently characterized by sub-ambient DSC to observe transitions of the formulated structures.

\section{Experimental Part}

\section{Materials}

Acrylamide/bis(acrylamide) (29/1) was obtained from Roche and used as received. 1,2-Bis(dimethylamino)ethane (TEMED) and ammonium persulfate (APS) were obtained from Sigma-Aldrich and stored in dark bottles before use. The separating buffer components including glycerol, sodium dodecylsulfate, and tris base were also obtained from Sigma-Aldrich and were formulated in solution based on a recipe from Stryer. ${ }^{[45]}$ The acidification of the buffer used $1 \mathrm{~N} \mathrm{HCl}$ (Sigma-Aldrich). The initial viscosity of these fluids was characterized as a function of composition by mixing the components without adding the curing agent, APS, which allowed for a longer working time to perform the frequency sweep measurements.

In assessing the dynamic viscosity during resin curing, the components were mixed adding APS as the last constituent. These were combined and mixed by moderate shaking followed by extracting fluid aliquots and transferring them to the rheometer to track transient viscosity.

\section{Methods}

\section{Rheological Assessment}

To determine the initial resin viscosity as a function of formulation, $1 \mathrm{~mL}$ of each mixture was deposited on the bottom surface of a TA Instruments ARES parallel plate rheometer, with a gap dimension of $1.5 \mathrm{~mm}$ and a radial dimension of $12.5 \mathrm{~mm}$. Measurements were taken in a strain controlled, dynamic sweep mode ranging between 1 and $15 \mathrm{rad} \cdot \mathrm{s}^{-1}$ using a frequency sweep increment of $0.05 \mathrm{rad} \cdot \mathrm{s}^{-1}$ with a strain of $200 \%$. At least three replicates were performed for each composition. Following these experiments, resins mixed with APS were analyzed to yield $\eta^{*}(t)$ which is what is reported here. The same hardware was used and a fixed frequency of $1 \mathrm{rad} \cdot \mathrm{s}^{-1}$ was continuously used with a strain of $5 \%$. The length of time between mixing the components together and initiating each test was also measured and added to the time of polymerization identified by the machine. All of these experiments were conducted under ambient conditions and we primarily report the complex viscosity, $\eta^{*}(t)$ measured during the dynamic cure experiments. 


\section{Differential Scanning Calorimetry}

Separately, once these mixtures were cured, samples were isolated and prepared for DSC. Samples were chilled to $-90^{\circ} \mathrm{C}$ and heated at a rate of $10^{\circ} \mathrm{C} \cdot \mathrm{min}^{-1}$ to $160^{\circ} \mathrm{C}$ using a TA Instruments Q-2000 DSC. From the sub-ambient analysis, the endotherm of water thawing in the gel was assessed as the temperature approached $0{ }^{\circ} \mathrm{C}$. The onset and peak temperatures were both determinant and the integration allowed the calculation of a heat of melting linked with the mass fraction associated with water in the gel.

\section{Results}

An example plot of the initial viscosity determination for $15 \%$ acrylamide gel (at $T=-10^{\circ} \mathrm{C}$ and with a strain of 200\%) devoid of the APS curing agent is shown in Figure 1. The slope of the linear response was the viscosity of the aqueous, pre-gel mixture. The pre-gel is clearly Newtonian, but the resolution of the torque response is not ideal. The variation in the viscosity of the initial formulations again devoid of APS is shown in Figure 2 as a linear function of composition of acrylamide in the pre-gel state.

The temperature dependence of viscosity, probed through a series of frequency sweeps, is shown in Figure 3. The presence of the salts in the buffer suppresses the freezing point of water in the mixture. The onset of freezing creates a slushy zone with a solid mass fraction that rises with further cooling. The Arrhenius activation barrier is observed as a rise in viscosity as temperature is lowered in the one-phase, liquid region.

Once APS was added to the acrylamide mixtures, rapid gelation ensued. The dynamic complex viscosity $\eta^{*}(t)$, of typical concentrations of separating gels used in electrophoresis was evaluated, but the concentration range was also expanded to resolve the boundaries of physical

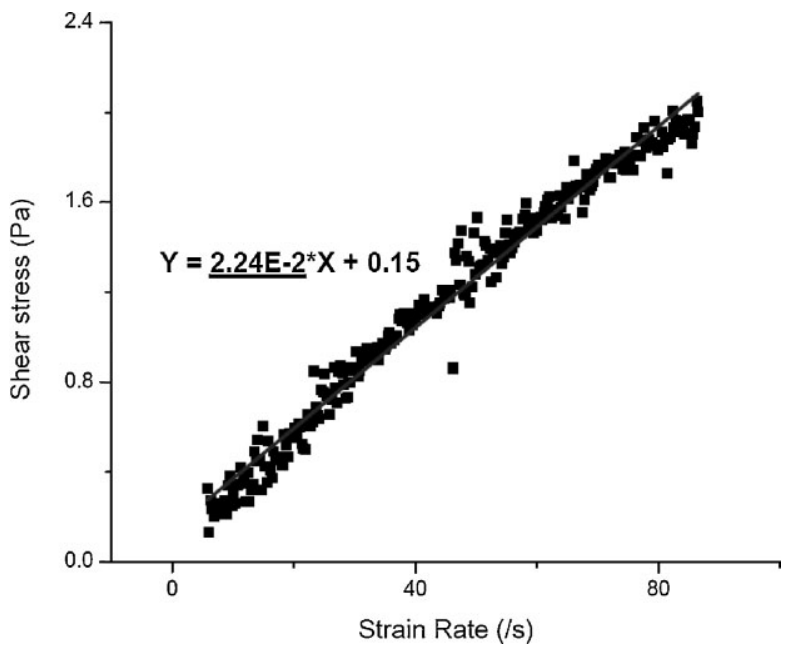

Figure 1. The $\eta_{\mathrm{o}}$ determination for $15 \mathrm{wt} . \mathrm{\%}$ acrylamide gel at $T=-10^{\circ} \mathrm{C}$ with a strain of $200 \%$.

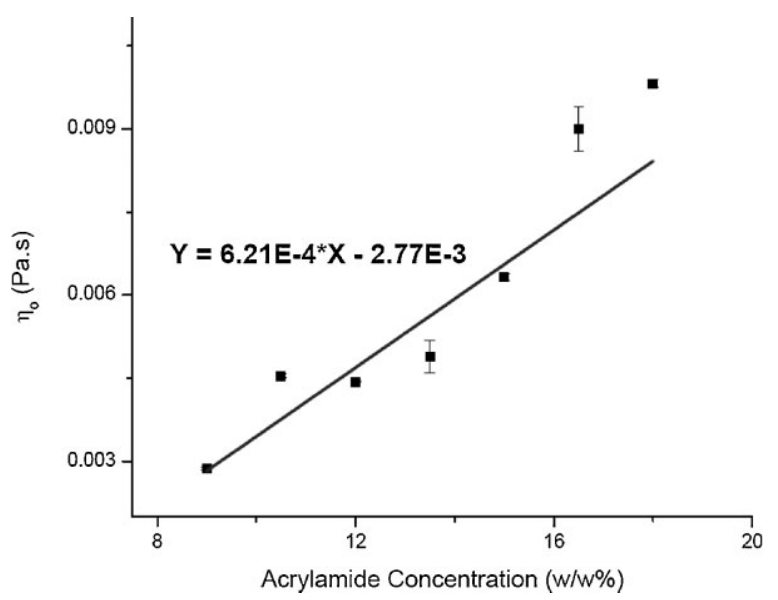

Figure 2. Variation of $\eta_{\mathrm{o}}$ at ambient temperature with acrylamide content in the aqueous formulation.

behavior associated with gelation. The results of $\eta^{*}(t)$, during conversion of three example compositions are shown in Figure 4. The cure time includes the machine time, the mixing time and the preparation time for the rheometer to initiate each test. A sigmoidal behavior is resolved in all conditions, with shorter induction times required to see a deviation from the initial viscosity at higher concentrations and a higher terminal condition associated with gel stiffness in the cured condition.

There is a problem with viscosity determinations at the early stages of the cure during these dynamic rheology experiments. $\eta_{0}$, determined by the frequency sweeps, is much lower than the instantaneous viscosity observed during the early stages of pre-cure dynamic measurement. The gel times are sufficiently short that sweeping through frequencies at each time point before gelation reduces the

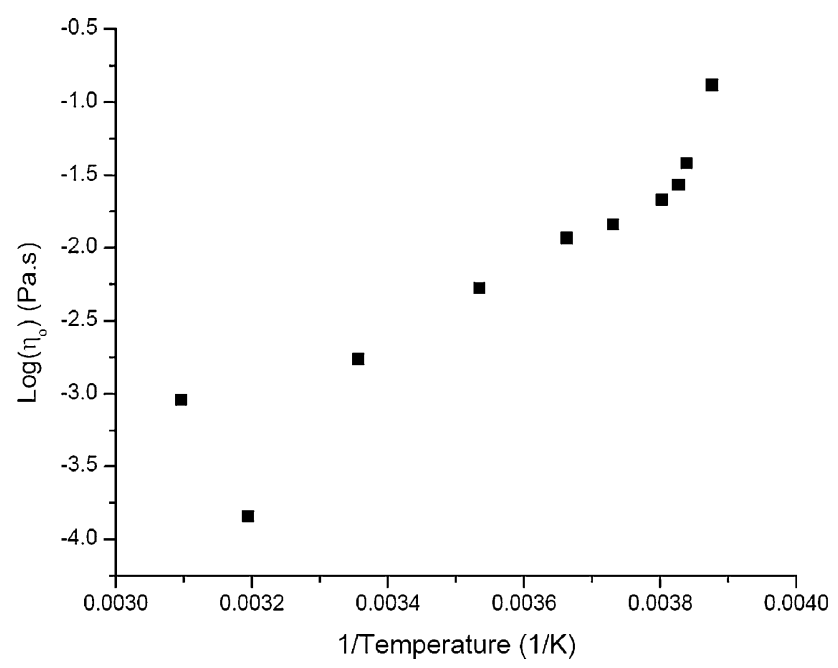

Figure 3. 15 wt.-\% acrylamide $\eta_{\mathrm{o}}(T)$ determined through frequency sweeps. Note the onset of water freezing marked by the slope discontinuity. 


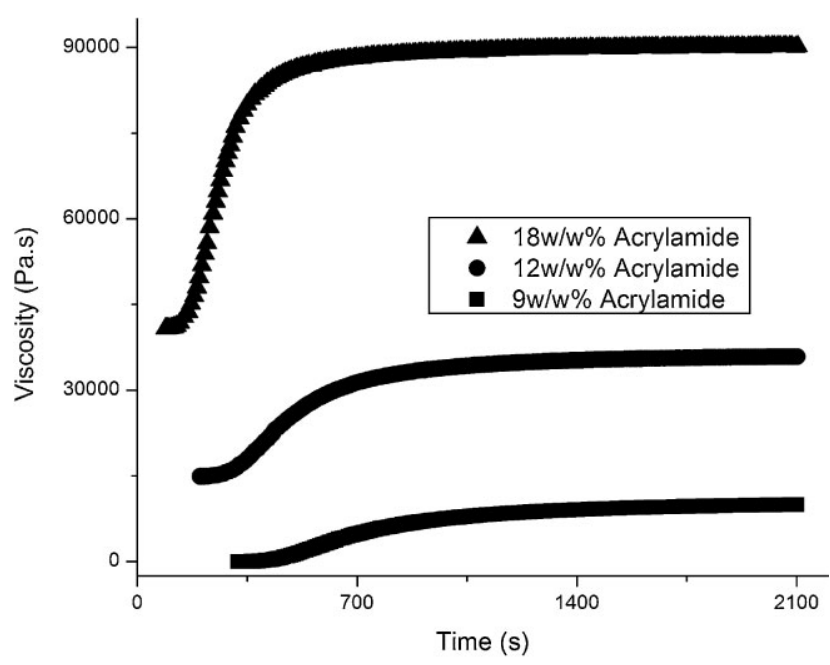

Figure $4 . \eta^{*}(t)$ during cure of 9,12 , and $18 \mathrm{wt} . \mathrm{-} \%$ aqueous acrylamide mixtures.

ability to resolve the transient region where viscosity is changing more dramatically. A more sensitive torque transducer would yield more accurate measurements of $\eta_{0}$ during the dynamic tests. More interesting is that as a function of formulated acrylamide content in the gels, the torque sensitivity can identify differences in the physical gel structure, with different plateau viscosities associated with the gel state. The trend in $\eta_{\infty}$ as a function of acrylamide composition follows a linear trend, shown in Figure 5. Even in a gel condition, these gels are compliant enough to resolve differences in network density as a function of composition by rheology.

The time scale of cure, occurring between 100 and $1000 \mathrm{~s}$ is similar to other work by Neamtu et al., ${ }^{[7,46]}$ Calvet et al., ${ }^{[1]}$ and Wang and Ugaz, ${ }^{[3]}$ and shorter than times for composite systems like those of Babaluo et al. ${ }^{[9,11]}$ with inert fillers included.

DSC results of a sample gel heated from an initial subambient cooled state are shown in Figure 6 . The endotherm below $0{ }^{\circ} \mathrm{C}$ is linked with the melting of the frozen water distributed within the gel. The size of the endotherm is proportional to the amount of water in the gel, the actual freezing point is depressed by aqueous impurities in the gel.

The calorimetric analysis results including the enthalpy and peak temperature of the melting endotherm associated with water in the gel, and the relative mass fraction of water freezing relative to pure water is included in Table 1.

There is a general trend that more water crystallizes out of the gels with lower acrylamide content. The molality of the gel solution is denoted by $m_{\mathrm{B}}$ and determined by dividing the freezing point depression by the cryoscopic constant for water. This molality shows a slight increasing trend associated with concentration. The variation in the peak temperature associated with this transition is very weakly dependent on the acrylamide concentration. This

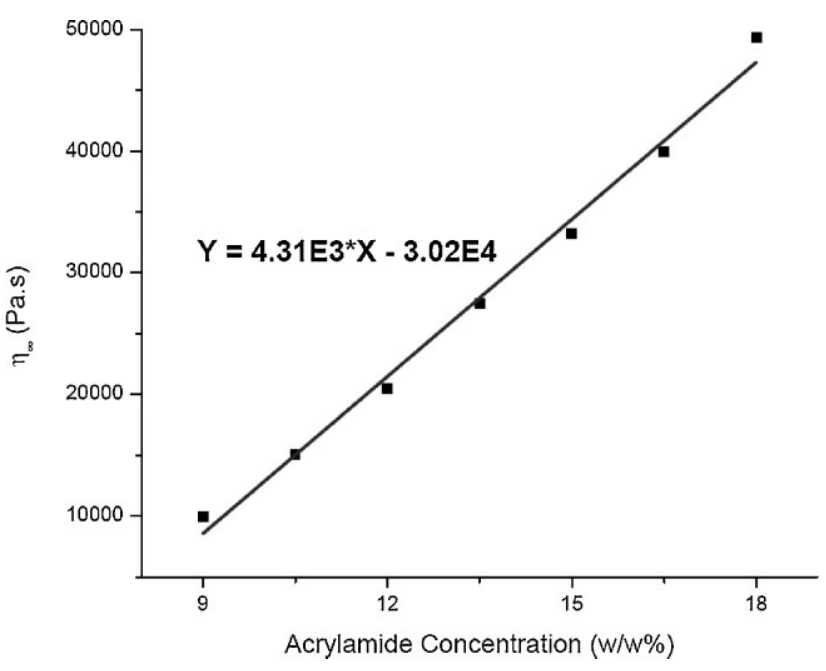

Figure 5. Variation in plateau viscosity in the cured state, $\eta_{\infty}$, with acrylamide content.

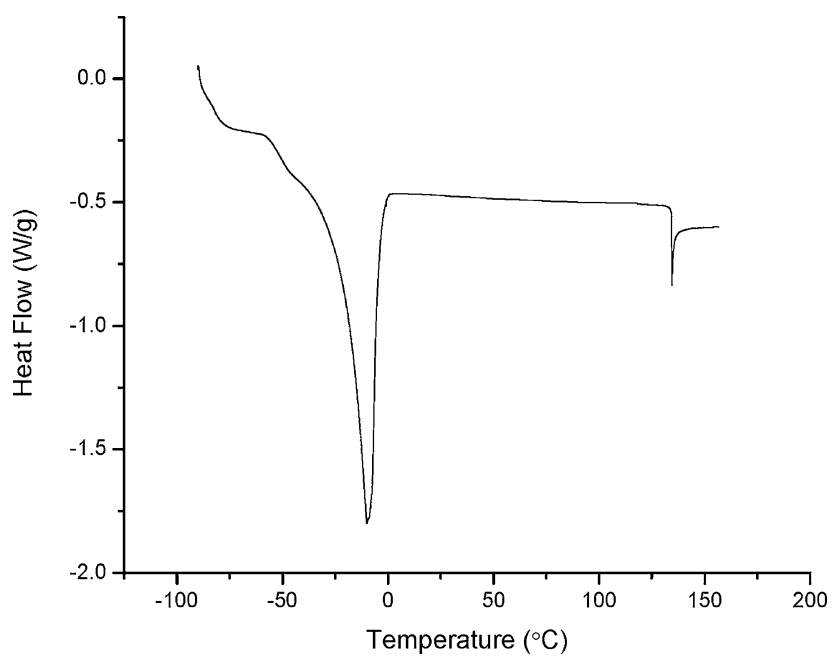

Figure 6. Representative DSC measurement of a cured $15 \mathrm{wt} .-\%$ acrylamide mixture.

general insensitivity is attributed to presence of the salt content in the buffer solution regardless of the acrylamide content contained in solution. The salts are much more effective at depressing the freezing point of water contained in the gel.

\section{Mathematical Analysis}

Armed with the dynamic viscosity results, it was desired to resolve whether simple viscosity advancement models could represent the self-cure gelation characteristics of these acrylamide gels. The results from the rheological experiments were inputted into Microcal Origin which has 
Table 1. Thermal analysis results for the acrylamide resins as a function of composition.

\begin{tabular}{|c|c|c|c|c|}
\hline $\begin{array}{l}\text { Acrylamide } \\
\text { content }\end{array}$ & Enthalpy & $\begin{array}{l}\text { Melting } \\
\text { fraction }\end{array}$ & $\begin{array}{c}\text { Peak } \\
\text { temperature }\end{array}$ & $m_{\mathrm{B}}$ \\
\hline wt.-\% & $J \cdot g^{-1}$ & acrylamide & ${ }^{\circ} \mathrm{C}$ & \\
\hline 9 & 151.7 & 0.455 & -8.58 & 4.6 \\
\hline 10.5 & 141.9 & 0.425 & -9.64 & 5.2 \\
\hline 12 & 140.3 & 0.421 & -9.73 & 5.2 \\
\hline 13.5 & 138.8 & 0.416 & -9.80 & 5.3 \\
\hline 15 & 135.3 & 0.406 & -9.89 & 5.3 \\
\hline 16.5 & 142.4 & 0.427 & -8.67 & 4.7 \\
\hline 18 & 143.2 & 0.430 & -7.67 & 4.1 \\
\hline
\end{tabular}

a data analysis package including a series of fitting routines. The sigmoidal analysis from Equation (1) was chosen, as was a power law model for further analysis to compare gel times for polymerization under the different formulation conditions. Example results for the two different analyses for the same resin are shown in Figure 7 and 8.

The power law analysis identified coefficients associated with the time dependent gelation. These coefficients are shown in Table 2 with the pre-gel exponent, $a$, and post-gel, $b$. The intersection of these two slopes yielded the gelation time, which is also shown as a function of acrylamide composition. The lower torque on the rheometer during the early, pre-gel stages raises some question as to the validity of first exponent. But the gelation response shows such a

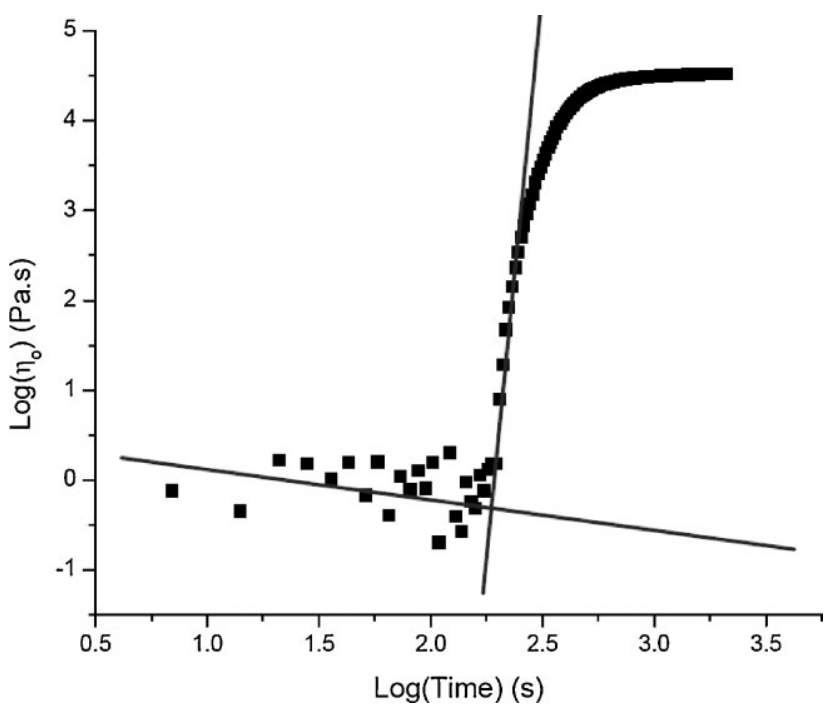

Figure 7. $\eta^{*}(t)$ of a 15 wt.-\% mixture cured at ambient temperature. The corresponding power law fit below and above the gel point also included.

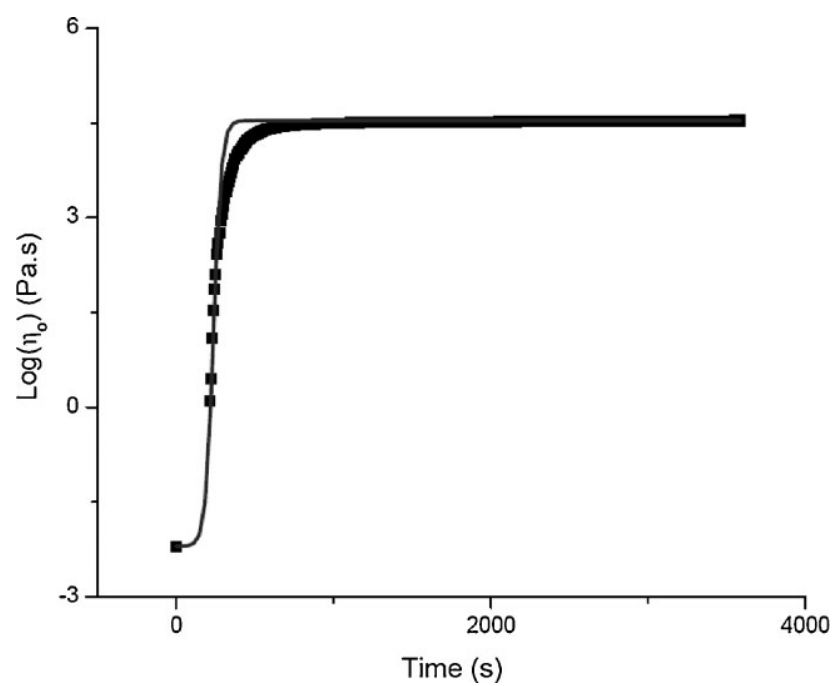

Figure 8 . The reduced $\eta^{*}(t)$ dataset for $15 \mathrm{wt}$.- $\%$ sample including the log-sigmoidal analysis.

Table 2. Power-law model parameters as a function of acrylamide formulation.

\begin{tabular}{lccc}
\hline $\begin{array}{l}\text { Acrylamide } \\
\text { content }\end{array}$ & $\boldsymbol{a}$ & $\boldsymbol{b}$ & $\boldsymbol{t}_{\text {gel }}$ \\
\cline { 1 - 2 } wt.-\% & & & $\mathbf{s}$ \\
\hline 9 & 0.05 & 17.8 & 345 \\
10.5 & 0.30 & 15.6 & 274 \\
12 & 0.28 & 15.2 & 220 \\
13.5 & -0.16 & 14.1 & 195 \\
15 & -0.08 & 16.0 & 196 \\
16.5 & -0.35 & 14.5 & 190 \\
18 & -0.22 & 11.7 & 127
\end{tabular}

marked rise that it is easily distinguishable from the pre-gel resin behavior. The rheology experiments clearly show a trend to shorter gel times at higher acrylamide concentrations. The variation in mixing between samples is a factor in the determination of these gel times.

The log-sigmoidal model was also used to represent the viscosity advancement. The viscosity of the final gel, a key variable in the log-sigmoidal model, was determined and included as a fixed constraint for each formulation. The initial viscosity varied as a function of formulation based on Figure 2 and was fixed at each condition. The presence of two time constants in the model allow for the capture of an appropriate induction time and the relative sharpness of the dynamic viscosity rise, the results of which are shown in Table 3.

Using only the datasets with $\log \eta^{*} \geq 0.5$ during the $\eta^{*}(t)$ measurements, the data was analyzed using the log- 
Table 3. Log-sigmoidal model parameters as a function of acrylamide formulation.

\begin{tabular}{lccc}
\hline $\begin{array}{l}\text { Acrylamide } \\
\text { content }\end{array}$ & $\log \left(\eta_{\mathbf{o}} / \mathrm{Pa} \cdot \mathbf{s}\right)$ & $\log \left(\eta_{\infty} / \mathrm{Pa} \cdot \mathbf{s}\right)$ & $\boldsymbol{t}_{\mathrm{gel}}$ \\
\cline { 1 - 3 } wt.-\% & & & $\mathbf{s}$ \\
\hline 9 & & & 314 \\
10.5 & -2.54 & 4.00 & 234 \\
12 & -2.32 & 4.18 & 201 \\
13.5 & -2.35 & 4.31 & 187 \\
15 & -2.31 & 4.44 & 185 \\
16.5 & -2.20 & 4.52 & 188 \\
18 & -2.01 & 4.60 & 119
\end{tabular}

sigmoidal model. The log-sigmoidal model contains two time constants to represent the datasets using Equation 1. To identify an appropriate gelation time, $t_{\mathrm{ge}}$, a relationship is needed to link the time constants to the gel time. We have previously reported on relationships to link these two characteristic times in terms of a physical time point like the gel time ${ }^{[47]}$ since $t_{0}$ corresponds to the midpoint of the viscosity change, and $4 \times \Delta t$ is equal to the duration of the dynamic range:

$$
t_{\mathrm{gel}}=t_{0}-2 \Delta t
$$

For the analysis, the initial viscosity at each composition was determined and fixed from the frequency sweep. The plateau viscosity was determined and fixed from the dynamic cure experiment. Removing the early-time viscosity measurements during the dynamic cure experiment associated with low resolution seen in Figure 7, the sigmoidal shape factors of the curves were modeled by fixing various $\Delta t$ values and determining $t_{0}$ for the best logsigmoidal fit seen in Figure 8. Once these were identified, Equation 2 was used to determine a simulated gel time based on the log-sigmoidal model identifying a time when the curve deviates from the initial viscosity; the results of which are shown in Table 3. Comparing the results from Table 2 and 3, the gel times between the two different models are close, although the power law model overestimates the gel time compared to the log-sigmoidal model at the lower concentrations of acrylamide. The power law and log-sigmoidal model determinations of gel times coincide much more closely when the acrylamide concentration is above $12 \%$.

Any appropriate rheological cure model would also include some sort of Arrhenius-like temperature dependence on the initial viscosity but since these experiments were conducted at room temperature, this is not considered as a part of the analysis here. With these gels, the complication of an aqueous phase transformation seen in Figure 3 suggests that a simple Arrhenius modification to the temperature dependent viscosity is inadequate.

\section{Conclusion}

From the work we have completed, the following conclusions can be made:

(i) increasing acrylamide composition shortens the working time of the evolving gel, and raises the initial viscosity and ultimate gel stiffness.

(ii) a small melting endotherm of ice melting in the frozen gel is observed, the size of which decreases as the acrylamide content is increased. There is a weak trend in the temperature associated with freezing point depression which is attributed primarily to the salt content in the buffer used in making the gel.

(iii) Mathematical analysis using both the power law and log-sigmoidal models has yielded an identified gel time in each case. The sigmoidal model also yields time constants associated with the dynamic viscosity advancement which control the shape of the viscosity advancement curve. The effect of changing composition on these time constants has been identified and expected trends of shorter working times with increased acrylamide concentration was observed.

(iv) The presence of higher concentration of acrylamide in the formulation allows for sufficiently reduced molecular mobility to reach a hindered state earlier in the time scale of polymerization, hence they have shorter time constants identified by both models.

Acknowledgements: T. S. acknowledges fellowship support through the Universite Claude Bernard (LYON1), France. B. J. L. and C. D. acknowledge internal support through the University of Michigan.

Received: July 22, 2009; Revised: September 18, 2009; Published online: December 17, 2009; DOI: 10.1002/mame.200900218

Keywords: gels; polyacrylamides; rheology; viscosity

[1] D. Calvet, J. Y. Wong, S. Giasson, Macromolecules 2004, 37, 7762.

[2] Y. Cohen, O. Ramon, I. J. Kopelman, S. Mizrahi, J. Polym. Sci., Part B: Polym. Phys. 1992, 30, 1055.

[3] J. Wang, V. M. Ugaz, Electrophoresis 2006, 27, 3349.

[4] A. M. Hecht, R. Duplessix, E. Geissler, Macromolecules 1985, 18, 2167.

[5] S. Kara, O. Pekcan, J. Appl. Polym. Sci. 2001, 80, 823.

[6] J. L. Trompette, E. Fabregue, G. Cassanas, J. Polym. Sci., Part B: Polym. Phys. 1997, 35, 2535. 
[7] I. Neamtu, L. E. Nita, A. P. Chiriac, M. Bercea, J. Optoelectron. Adv. Mater. 2006, 8, 201.

[8] I. Neamtu, L. E. Nita, A. P. Chiriac, M. Bercea, "Rheological Monitoring of In situ Poly(acrylamide) Gel Preparation", in: 6th International Balkan Workshop on Applied Physics, Constanta, Romania 2005, p. 201.

[9] A. A. Babaluo, M. Kokabi, A. Barati, J. Eur. Ceram. Soc. 2004, 24, 635.

[10] A. Barati, M. Kokabi, M. H. N. Famili, Iran. Polym. J. 2003, 12, 127.

[11] M. Kokabi, A. A. Babaluo, A. Barati, J. Eur. Ceram. Soc. 2006, 26, 3083.

[12] M. Tahmasebpour, A. A. Babaluo, S. Shafiei, E. Pipelzadeh, Powder Technol. 2009, 191, 91.

[13] M. Tahmasebpour, A. A. Babaluo, M. K. R. Aghjeh, J. Eur. Ceram. Soc. 2008, 28, 773.

[14] T. M. Klaveness, P. Ruoff, J. Kolnes, J. Phys. Chem. 1995, 99, 8255.

[15] C. Gelfi, P. G. Righetti, Electrophoresis 1981, 2, 213.

[16] C. Gelfi, P. G. Righetti, Electrophoresis 1981, 2, 220.

[17] P. G. Righetti, C. Gelfi, A. B. Bosisio, Electrophoresis 1981, 2, 291.

[18] G. Patras, G. G. Qiao, D. H. Solomon, Electrophoresis 2001, 22, 4303.

[19] E. S. Matsuo, M. Orkisz, S. T. Sun, Y. Li, T. Tanaka, Macromolecules 1994, 27, 6791.

[20] S. Takata, T. Norisuye, M. Shibayama, Macromolecules 1999 , 32, 3989.

[21] F. Teyssandier, Y. Y. Sun, C. P. Wong, B. J. Love, Macromol. Mater. Eng. 2008, 293, 828.

[22] F. Teyssandier, M. Ivankovic, B. J. Love, J. Appl. Polym. Sci., 2010, 115, 1671.

[23] B. J. Love, F. Teyssandier, Y. Y. Sun, C. P. Wong, Macromol. Mater. Eng. 2008, 293, 832.

[24] B. J. Love, Part. Sci. Technol. 2004, 22, 285.

[25] B. J. Love, F. Piguet-Ruinet, J. Appl. Polym. Sci. 2007, 106, 3605.
[26] B. J. Love, F. Piguet-Ruinet, F. Teyssandier, J. Polym. Sci., Part B: Polym. Phys. 2008, 46, 2319.

[27] F. Teyssandier, B. J. Love, unpublished.

[28] D. S. Achilias, Macromol. Theory Simul. 2007, 16, 319.

[29] D. S. Achilias, C. Kiparissides, Macromolecules 1992, 25, 3739.

[30] Y. Y. Sun, Z. Q. Zhang, C. P. Wong, IEEE Trans. Compon. Packag. Technol. 2006, 29, 190.

[31] Y. Y. Sun, Z. O. Zhang, C. P. Wong, Macromol. Mater. Eng. 2005, 290, 1204.

[32] M. Cioff, K. J. Ganzeveld, A. C. Hoffmann, L. Janssen, Polym. Eng. Sci. 2004, 44, 179.

[33] M. Cioffi, K. J. Ganzeveld, A. C. Hoffmann, L. Janssen, Polym. Eng. Sci. 2002, 42, 2383.

[34] M. Cioffi, A. C. Hoffmann, L. Janssen, Polym. Eng. Sci. 2001, 41, 595.

[35] H. Haddadi, M. H. N. Famili, E. Nazokdast, S. Moradi, Iran. Polym. J. 2006, 15, 967.

[36] C. Y. M. Tung, P. J. Dynes, J. Appl. Polym. Sci. 1982, 27, 569.

[37] A. Apicella, L. Nicolais, M. Iannone, P. Passerini, J. Appl. Polym. Sci. 1984, 29, 2083.

[38] F. Y. C. Boey, W. Qiang, J. Appl. Polym. Sci. 2000, 76, 1248.

[39] H. H. Winter, Polym. Eng. Sci. 1987, 27, 1698.

[40] L. Matejka, Polym. Bull. 1991, 26, 109.

[41] E. Mounif, V. Bellenger, A. Tcharkhtchi, J. Appl. Polym. Sci. 2008, 108, 2908.

[42] Y. S. Yang, L. Suspene, Polym. Eng. Sci. 1991, 31, 321.

[43] A. Y. Malkin, S. G. Kulichikhin, M. L. Kerber, I. Y. Gorbunova, E. A. Murashova, Polym. Eng. Sci. 1997, 37, 1322.

[44] P. J. Halley, M. E. Mackay, Polym. Eng. Sci. 1996, 36, 593.

[45] L. Stryer, Biochemistry, Freeman, New York 1995.

[46] L. E. Nita, A. P. Chiriac, M. Bercea, I. Neamtu, "In situ Monitoring the Sol-Gel Transition for Polyacrylamide gel", in: 3rd Annual European Rheology Conference, Hersonissos, Greece 2006, p. 595.

[47] F. Teyssandier, B. J. Love, Polym. Eng. Sci. 2010 DOI: 10.1002/ pen.21560. 